\title{
Water Quality Monitoring System Using IOT
}

\author{
Suruchi Pokhrel ${ }^{1}$, Anisha Pant ${ }^{1}$, Ritisha Gautam ${ }^{1}$, and Dinesh Baniya Kshatri ${ }^{1}$ \\ ${ }^{1}$ Department of Electronics and Computer Engineering, Thapathali Campus, Institute of Engineering, \\ Tribhuvan University, Kathmandu, Nepal \\ "Corresponding Email: pokhrelsuruchi@gmail.com
}

\begin{abstract}
Water pollution is one of the growing issues in a developing country like Nepal. In the present scenario, we are usually thoughtlessly trusting the drinking water suppliers with our health. Even though the water is purified as well as checked in the central distribution systems, the supplier, along with the general public is unaware of the water quality that reaches the end-users. By focusing on these above issues, we propose a low-cost monitoring system that can monitor water quality such as $\mathrm{pH}$ (potential of Hydrogen) and conductivity on a timely basis using the Internet of Things. The water quality monitoring sensors sense the necessary physical parameters and convert them into equivalent electrical form, i.e. by providing certain voltage as an output corresponding to the respective physical quantity. This value is mapped to the respective water quality measure and is stored in a database through the microcontroller using the Internet of Things. This aids the suppliers to centralize the regular monitoring of water from various locations as well as the supply pure water to the end-users.
\end{abstract}

Keywords: Conductivity, Internet of Things, Potential of Hydrogen, Water Quality Monitoring

\section{INTRODUCTION}

Water covers $71 \%$ of the earth's surface and is vital for all known forms of life. Only $2 \%$ of this water is freshwater and even so, only $0.036 \%$ of it is accessible for use. [1] Having said that, we can conclude that the availability of water is extremely limited. Due to this, it is essential that we make the best use of it by making sure that the water available to use is pure in all forms.

Water quality monitoring is a crucial task that ensures that the water we consume fills the necessary standard. This includes observing parameters such as $\mathrm{pH}$ (potential of Hydrogen), Turbidity, Hardness, Color, amount of Iron and Ammonia. It is particularly vital in a nation like Nepal where the surface water has been deteriorating due to urbanization especially in cities like Kathmandu. Even though the residents here rely upon Kathmandu Upatyaka Khanepani Limited for their daily supply of drinking water, it is no longer dependable as there were 6278 cases of typhoid, 10,392 cases of diarrhea, 583 cases of jaundice in the year 2074 and 2075. There were a total of 23,342 cases of water-borne diseases and 78 of them were even fatal [2]. 
The existing system of water quality monitoring in these suppliers is based upon Water Sampling, testing samples and analyzing the results. This becomes a tedious and expensive task which does not ensure the consistent supply of suitable water at the end-users. According to the Kathmandu Valley Water Supply Management Board, out of 460 registered drinking water supply tankers, only 14 of them got the approval sticker [3].

To counteract the given issues, our proposed system is a wireless water quality monitoring system that measures two of the important parameters, $\mathrm{pH}$ and electrical conductivity on a certain time basis. These measurements are converted into respective units and sent to the database using IoT for further inspection. The parameters in the database are compared with the standard required for the pure water (6.5 to 7.8 for $\mathrm{pH}$ and $0-50 \mathrm{mS}$ ) for electrical conductivity [4]. The system alerts the user of any deviation from the standard value and also suggests the suitable remedies to restore the water quality.

For further details, section II explains the related works that have been done in this field, section III provides explains the implemented methodology, section IV focuses on the output of the system while section $\mathrm{V}$ illustrates the analysis of the results.

\section{RELATED WORKS}

Water quality, through the past decade, has been monitored through tedious processes of collecting samples from the water resources and testing if their physical, chemical as well as bacteriological properties meet the standard for the designated requirements in the laboratory. This method, despite being accurate, does not take into account the real time quality of water, and is also more time consuming. In recent years, more convenient approaches have been applied to tackle these drawbacks.

Pradeep Kumar Somasundara and Dharon Joseph Ediosn, proposed the use of RF module and IoT in order to constantly monitor the water which is available through taps through various sensors and transmit the data available to a remote base station. [5].Shruti Shridharan illustrated the use of Wireless Sensor Network (WSN) for monitoring the quality of water in the water resources and analyzing its usability for the domestic purposes [6].Pandian D R and Dr. Mala K proposed a "smart" water quality monitoring device that makes the use of IoT to send the measured water quality parameters to their nearby local testing laboratory [7].A.N.Prasad et al. investigated four of such factors that are $\mathrm{pH}$, oxidation and reduction potential (orp), conductivity and temperature using a Remote Sensing (RS) technology. only be sending data in case the values are just a false alarm [8].S.Suganya et al. proposed the use of IoT based water purity measuring system for industrial purposes that makes the use of GSM module in order to send the message to an industrial visitor in case of an abnormality in the water sources. [9].

\section{METHODOLOGY}

Different systematic, theoretical and scientific analysis were performed to accomplish the project. System architecture was built and then implemented to achieve the specific goal.

\subsection{SYSTEM ARCHITECTURE}

The block diagram of the proposed system is shown in the figure above. It shows the entire connectivity of the system. The major components of the Water Quality Monitoring system are the sensors, the internet and the microcontroller. Our project uses ESP32 as the control device that easily connects to existing Wi-Fi network \& creates a Web Server. We are going to configure our ESP32 into Station (STA) mode and create a webserver to serve up web pages to any connected client under existing network. The sensors used in our project are $\mathrm{pH}$ and conductivity. These sensors are dipped in water which gives the corresponding PH, conductivity value based on Water Quality in which sensors are deployed. The 
sensors are interfaced with the ESP32 by writing a piece of code in Arduino IDE and uploading to the ESP32. The system is centralized through the microcontroller. The micro-controller is responsible for controlling all the devices connected to it. The communication between the server and ESP32 is done via Wi-Fi protocol 802.11/b

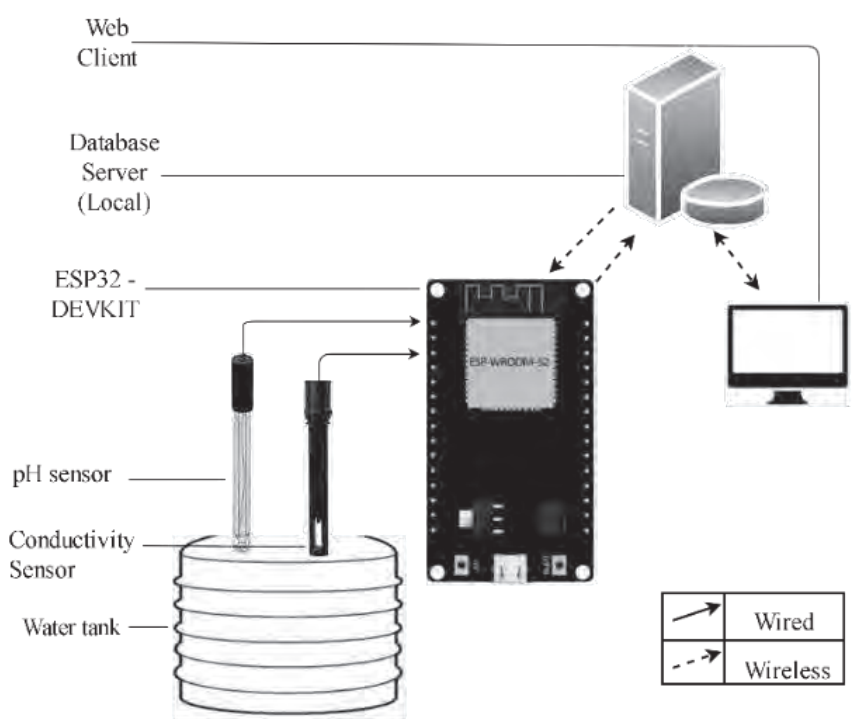

Figure 1: System Architecture

The data in the microcontroller are send to the webserver by HTTP (Hypertext Transfer Protocol) methods. The HTTP is designed to enable communications between clients and servers. HTTP works as a request-response protocol between a client and server. There are different HTTP methods like POST, GET, PUT, DELETE etc. for different purposes. Among which we use POST to send data to a server to create/update a resource. A webserver is simply a server that gives web services, handles the web requests, give responses and follows HTTP protocol.

The data is stored in the database from the web server. We are using MongoEngiene Library as a database adapter which is an Object-Document Mapper (ODM) for working with MongoDB from python. The data from the server is obtained by GET method. The GET method requests a representation of the specified resource. Requests using GET retrieves data and the data are passed into the html by the use of the jinja template. We are using ngrok as a tunneling tool. Ngrok allows us to expose a web server running on our local machine to the internet. We just need to specify what port our web server is listening to. Ngrok tunnels web socket connections over HTTP tunnels without any change and works everywhere even when device changes networks.

The data stored in the database is then displayed in the web page in a tabular format. The web page helps monitor the $\mathrm{pH}$ and turbidity and alerts the user of any mishaps. Also, in case of any deviation, the possible remedies in order to restore its quality is also displayed. 


\subsection{INSTRUMENTATION}

Various instruments have been used in order to sense the physical parameters and wirelessly transmit the sensed parameters.

\subsection{1. pH SENSOR}

$\mathrm{pH}$ is the measure of relative amount of free hydrogen and hydroxyl ions in water. $\mathrm{pH}$ is so closely associated with other aspects of water quality. $\mathrm{pH}$, in exact meaning is the potential of Hydrogen. It gives the measure of how acidic or alkaline any liquid is which ranges from 0 to 14, where less than 7 represents acidic and more than 7 represents alkaline. Consuming acidic or alkaline water is very harmful, and the $\mathrm{pH}$ of drinking water must lie within 6.5 to $7 . \mathrm{A} \mathrm{pH}$ sensor is an instrument that measures the hydrogen-ion activity in water-based solutions, indicating its acidity or alkalinity expressed as $\mathrm{pH}$. $\mathrm{pH}$ sensor is similar as a traditional $\mathrm{pH}$ meter with the additional advantages of automated data collection, graphing and data analysis. $\mathrm{pH}$ sensor is made up of four components which includes a measuring electrode, a reference electrode, preamplifier and transmitter or analyzer.

$\mathrm{pH}$ sensor basically works on the fact that interface of two liquids produces an electric potential which can be measured. In other words when a liquid inside an enclosure made of glass is placed inside a solution other than that liquid, there exists an electrochemical potential between the two liquids.

\subsubsection{CONDUCTIVITY SENSOR}

Electrical conductivity is the measure of water's capability to conduct electricity, which is directly related to concentration of salts in water. It estimates the total amount of solids dissolved in water. Water that is high in sodium dissolved is unsuitable for drinking by individuals who have difficulty metabolizing high levels of sodium, such as some people suffering from cardiac disease or toxemia of pregnancy.

Two electrodes are positioned opposite from each other. An AC voltage is applied to the electrode, which generates current in the medium. The cations move to the negatively charged electrode while the anions move to the positively charged electrode. The more, free charge carriers the liquid contains, the higher the electrical conductivity and the current flow. Pure water is bad conductors since they contain only few ions.

\subsubsection{ESP-32}

The ESP32 is a dual core, $180 \mathrm{MHz}$ which is highly integrated with in-built antenna switches, RF balun, power amplifier, low noise receiver amplifiers, filters and power management modules. ESP32 can interface with other systems to provide Wi-Fi and Bluetooth functionality through its SPI(Serial Peripheral Interface)/SDIO( Secure Digital Input Output) or I2C(Inter-Integrated Circuit)/ UART(Universal Asynchronous Receiver/Transmitter) interfaces. It has been designed for mobile, wearable electronics and Internet of Things applications with the aim of achieving the lowest power consumption with a combination of several proprietary techniques. This is a successor microcontroller of the previously manufactured ESP8266. While both of these microcontrollers support Wi-Fi the major advantage of ESP32 is that it has 36 GPIO (General-purpose Input Output) pins compared to the 17 pins in the ESP8266. This 32-bit processor has 12 ADC channels and 16 PWM(Pulse Width Modulation) channels. With its GPIO being around four times faster than the ESP8266, ESP 32 also additionally comes with touch sensitive pins to wake the processor on from deep sleep as well as a built-in temperature sensor. Since it can perform as a complete standalone system or as a slave device to a host MCU(Microcontroller Unit), this reduces our requirement for an extra microcontroller such as Arduino Uno, unlike the ESP-8266. Hence, the single microcontroller replaces our need for any other host microcontroller and the Wi-Fi module to interact with the internet. 


\subsection{SOFTWARE AND FRAMEWORKS}

Specific software were used to accomplish the project accuracy in a quick, efficient and effective way of data analysis, storage and visualization.

\subsubsection{Mongo DB}

MongoDB is a cross platform and open source database management system (DBMS) that uses a document-oriented database model which supports various forms of data. It is one of numerous nonrelational database technologies which arose in the mid-2000s under the NoSQL(Structured Query Language) banner for use in big data applications and other processing jobs involving data that doesn't fit well in a rigid relational model. Unlike in relational databases which employs the use of rows and columns, the MongoDB consists of collections and documents. The documents are stored in JSON(JavaScript Object Notation) or BSON(Binary JSON ) format. Documents of the MongoDB are just like the JSON objects but they instead have BSON format which accommodates more data types. MongoDB follows a highly flexible type of data model that that allows to combine and store data of multivariate type.

\subsubsection{ARDUINO IDE}

Arduino IDE is an open source cross platform application that is used to write and upload programs to the microcontrollers that are compatible with the Arduino. It runs on Windows, Mac OS X and Linux and is written in Java programming language. The main code here, is known as sketch and this code, when created in the IDE platform generated the Hex file. This hex file is the actual file which is transmitted to the microcontroller board. It supports both the $\mathrm{C}$ as well as $\mathrm{C}++$ languages. In our project, we use this software to program our ESP32 microcontroller, which replaces our need for any other microcontrollers.

\section{RESULTS}

The proposed system accepts the data from sensor and identify the deviation in water quality parameter from predefined set of standard value and display the data in hourly time basis using wireless communication. The user is displayed with the interface where one can view the recorded values of the different parameters of desired time period. The deviation of the parameters is also be displayed in a graph in daily basis. With the data analysis, instant results are displayed in the web browser anytime and anywhere. However, the efficiency of the system cannot be guaranteed due to various unavoidable errors of the equipment. Despite of the error factors, we expect to draw the best performance out of this system. The system can prove its worth by delivering accurate and consistent data throughout the testing period and with the added feature of incorporating IoT platforms for real time water monitoring, this system can be an excellent contender in real time water monitoring. 


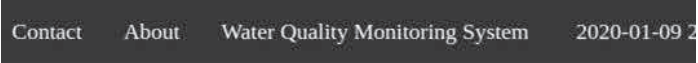

\section{Water Quality Monitoring System}

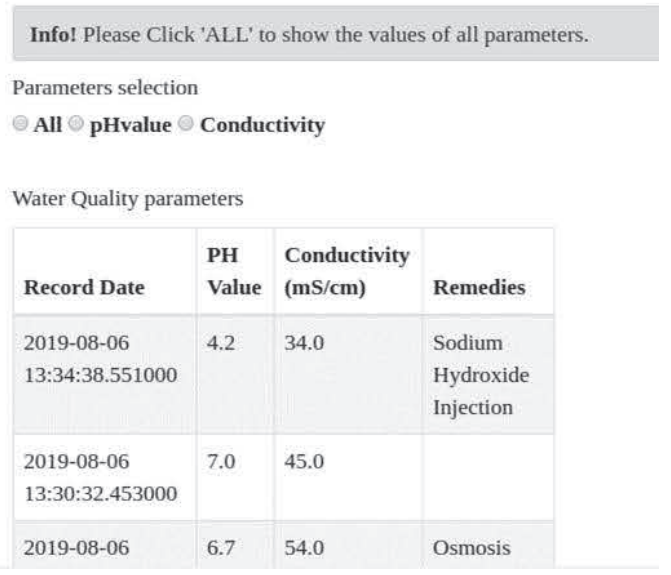

Figure 2: Webpage showing all parameters along with remedies

Given above is the user interface of the system when the user logs into the specific web page. The user is initially shown the records of both the $\mathrm{pH}$ and conductivity parameters. Here the records are shown in the time interval of 30 seconds. In case of any deviation from the standard parameters like shown above, the remedies are also displayed in order to inform the user as to what can be done in order to purify the water.

\begin{tabular}{|l|l|}
\hline Record Date & PH Value \\
\hline 2019-08-06 13:08:01.661000 & 3.0 \\
\hline 2019-08-06 13:13:07.991000 & 11.3 \\
\hline 2019-08-06 13:18:14.075000 & 13.0 \\
\hline 2019-08-06 13:22:20.119000 & 18.35 \\
\hline 2019-08-06 13:26:26.227000 & 6.7 \\
\hline 2019-08-06 13:30:32.453000 & 7.0 \\
\hline $2019-08-06$ 13:34:38.551000 & 4.2 \\
\hline
\end{tabular}

Figure 3: Showing $\mathrm{pH}$ value only 


\begin{tabular}{|l|l|}
\hline Record Date & $\begin{array}{l}\text { Conductivity } \\
\text { (mS/cm) }\end{array}$ \\
\hline 2019-08-06 13:08:01.661000 & 45.0 \\
\hline 2019-08-06 13:13:07.991000 & 23.0 \\
\hline 2019-08-06 13:18:14.075000 & 28.0 \\
\hline 2019-08-06 13:22:20.119000 & 0.05 \\
\hline 2019-08-06 13:26:26.227000 & 54.0 \\
\hline 2019-08-06 13:30:32.453000 & 45.0 \\
\hline 2019-08-06 13:34:38.551000 & 34.0 \\
\hline
\end{tabular}

Figure 4: Showing Conductivity value only

The user can also choose to view only of the two parameters. In that case the user also has an option of viewing a graphical form of the deviation of the specific parameters with time which makes the monitoring more interactive.

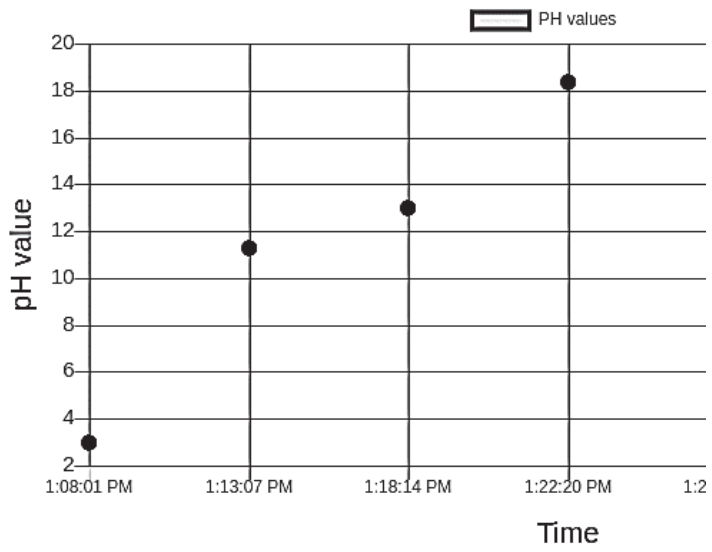

Figure 5: Graph showing $\mathrm{pH}$ deviation every 5 minutes

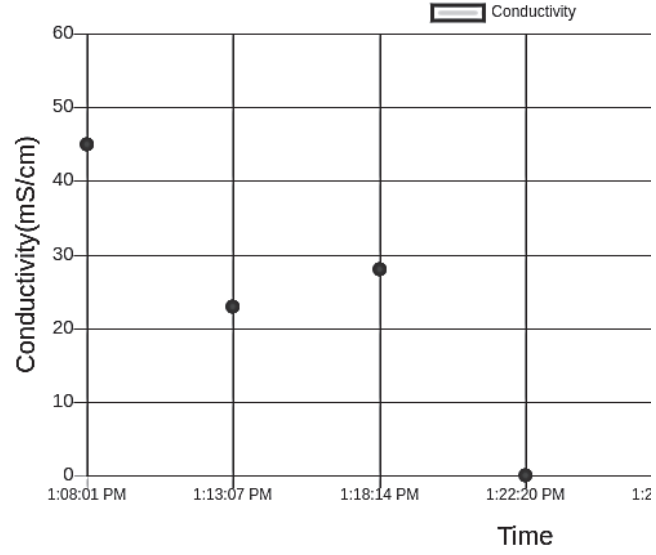

Figure 6.Graph showing conductivity every 5 minutes

\section{ANALYSIS}

The result obtained from the system is then analyzed and compared with the standard value along with its error calculation. This is a very crucial step in order to make the system more accurate.

\subsection{PH SENSOR RESULT ANALYSIS}

Table 1: Output Digital Values for Respective Solution

\begin{tabular}{|c|c|c|c|c|}
\hline Solution & Std. pH & Voltage Counts & Corresponding voltage & Obtained pH \\
\hline Distilled water & 7 & 2595 & $3.23 \mathrm{~V}$ & 7.2 \\
\hline
\end{tabular}

Output of the $\mathrm{pH}$ sensor for distilled water found to be 7.2. Since distilled water is neutral with $\mathrm{pH}$ exactly 7 (at 293K) [10] so there was some error while measuring $\mathrm{pH}$. The probe of the $\mathrm{pH}$ sensor with 
which $\mathrm{pH}$ is measured is very sensitive. It even reacts to a very small drop of liquid molecule in its probe. The sensor is very sensitive to the environment. Since the parameters of water is defined in molecule level, the shaking of the $\mathrm{pH}$ probe would cause the molecules to deviate from its position thereby affecting ph.

Error calculation for $\mathrm{pH}$ :

$$
\begin{gathered}
\text { Error } \%=\frac{\mid \text { measured value }- \text { std.value } \mid}{\text { std.value }} * 100 \% \\
=\frac{|7.2-7|}{7} * 100 \% \\
=2.85 \%
\end{gathered}
$$

Hence the error calculation of $\mathrm{pH}$ sensor for distilled water is $2.85 \%$

\subsection{CONDUCTIVITY SENSOR OUTPUT ANALYSIS}

Table 2: Table Showing rainwater digital output

\begin{tabular}{|l|l|l|l|}
\hline Solution & Standard Conductivity & Obtained Conductivity & Digital Output \\
\hline Rainwater & 50 & 50.2 & 567 \\
\hline
\end{tabular}

Error calculation for Conductivity:

$$
\begin{gathered}
\text { Error } \%=\frac{\mid \text { measured value }- \text { std.value } \mid}{\text { std.value }} * 100 \% \\
=\frac{|52.8-50|}{50} * 100 \% \\
=5.6 \%
\end{gathered}
$$

Hence the error calculation of $\mathrm{pH}$ sensor for distilled water is $5.6 \%$. The output of conductivity sensor for Rain water was found to be $52.8 \%$. Since rain water is neutral, its conductivity should be $50 \mu \mathrm{S} / \mathrm{cm}$ (at 293K) [11].So there were some error while making conductivity sensor. Conductivity sensor is very sensitive which even make differences its output if the probe is moved as molecules move apart changing the conductivity value. Also, the nichrome wire used for making the conductivity sensor is of 32 gauage which also makes difference in the measured value.

\section{CONCLUSION}

This Project demonstrates a smart water quality monitoring system using IoT to validate the system measurement accuracy. In this project, we propose a system that integrates with the IoT technology for real-time water quality monitoring. The system can monitor water quality automatically, and it is low in cost and does not require more manpower. This real-time application generates, collects, transfers, and stores sensor data in the web server. Data analysis was done, and instant reports were generated to display from anywhere and anytime in the web browser. This system was designed to reduce manpower, lower cost, and increase efficiency in water distribution and monitoring. Experiments affirm the good performance of the proposed system. In a nutshell, the system has proved its worth by delivering accurate and consistent data throughout the testing period and with the added feature of incorporating IoT platforms 
for real time water monitoring, this should be an excellent contender in real time water monitoring solutions. Therefore, this proposed system is reliable for a real environment deployment.

\section{FUTURE ENHANCEMENTS}

The current System illustrates the design and performance of a real-time monitoring system for measuring the water quality of two parameters of water. The reconfigurable system can be further improved by a combination of more sensors for the large measuring area. Additionally, the alarm system unit can be deployed in the system when there occurs large deviation in parameter values from the standard one along. The system has good flexibility. Only by replacing the corresponding sensors and changing the relevant software programs, this system can be used to monitor other water quality parameters.

The system can be expanded to monitor hydrologic, air pollution, soil pollution and so on. It has widespread application and extension value. By keeping the embedded devices in the environment for monitoring enables self-protection (i.e. smart environment) to the environment. To implement this method, it is necessary to deploy the sensor devices in the environment for collecting the data and analysis. By deploying sensor devices in the environment, we can bring the environment into real life i.e. it can interact with other objects through the network. Then the collected data and analysis results will be available to the end user through the Wi-Fi. Mobile application can be made to see the measured value of the water parameters and the use of GSM module will send the message to the respective user about the measured water parameter value.

\section{ACKNOWLEDGMENT}

The authors would like to express their deepest gratitude to the Department of Electronics and Computer Engineering of the Institute of Engineering, Thapathali Campus for providing access to laboratory equipment to conduct this study. The authors are also indebted to the Robotics and Automation Center also belonging to the Institute of Engineering, Thapathali Campus for the sharing of technical experience and facilitating the procurement of hardware instruments required to conduct this study.

\section{REFERENCES}

[1] I. A. Shiklomanov, Water in Crisis, New York: Oxford University Press, 1993.

[2] Department of Health Services, "Annual Report 2074/75," Government of Nepal, Kathmandu, 2018.

[3] thewaterproject.org, "Water Crisis In Nepal," June 2017. [Online]. Available: https://thewaterproject.org/water-crisis/water-in-crisis-nepal.

[4] World Health Organization(WHO), "pH in Drinking Water," 2007.

[5] P. K. Somasundaram and D. J. Ediosn, "Monitoring Water Quality using RF Module," International Journal of Application or Innovation in Engineering \& Management (IJAIEM), vol. 2, no. 7, pp. 220-224, 2013.

[6] S. Shridharan, "Water Quality Monitoring System Using Wireless Sensor Network," International Journal of Advanced Research in Electronics and Communication Engineering (IJARECE), pp. 399-402, 2014. 
[7] D. M. K. Pandian D R, "Smart Device to monitor water quality to avoid pollution in IoT environment," International Journal of Emerging Technology in Computer Science \& Electronics (IJETCSE), vol. 12, no. 2, pp. 120-125, 2015.

[8] K. A. M. F. R. I. H. H. A.N.Prasad, "Smart Water Quality Monitoring System," in Asia-Pacific World Congress on Computer Science and Engineering (APWC on CSE), Osaka, Japan, 2015.

[9] K. D. G. ,. S. Suganya, "Iot based Standard Water Measuring System using GSM," IJRASET, vol. 6, no. 4, pp. 74-78, 2018.

[10] K. Kulthanan, P. Nuchkull and S. and Varothai, "The pH of water from various sources: an overview for recommendation for patients with atopic dermatitis," Asia Pac Allergy., vol. III, no. 3, pp. 155-160, 2013.

[11] Clean Water Team (CWT), "Electrical conductivity/salinity Fact Sheet, FS3.1.3.0(EC).," California State Water Resources Control Board (SWRCB), Sacramento, CA, 2004. 